\title{
Long Noncoding RNA PVT1 Predicts Poor Prognosis and Promotes the Progression of Colorectal Cancer Through the MiR-24-3p/NRP1 Axis in Zebrafish Xenografts
}

\section{Hailin Yin}

Lianshui County People's Hospital, Kangda College of Nanjing Medical University

\section{Shanye Gu}

Lianshui County People's Hospital, Kangda College of Nanjing Medical University

\section{Guiqin Li}

Lianshui County People's Hospital, Kangda College of Nanjing Medical University Hanxu Yu

Lianshui County People's Hospital, Kangda College of Nanjing Medical University

\section{Xishan Zhang}

Lianshui County People's Hospital, Kangda College of Nanjing Medical University

\section{Yangsong Zuo ( $\square$ zys126@njmu.edu.cn )}

Lianshui County People's Hospital, Kangda College of Nanjing Medical University

\section{Research Article}

Keywords: colorectal cancer, IncRNA PVT1, prognosis, miR-24-3p, NRP1, zebrafish xenograft

Posted Date: February 28th, 2022

DOI: https://doi.org/10.21203/rs.3.rs-1353449/v1

License: (c) (i) This work is licensed under a Creative Commons Attribution 4.0 International License.

Read Full License 


\section{Abstract}

\section{Background}

Long noncoding RNAs (IncRNAs) play important roles in the progression of human cancer. It is reported IncRNA plasmacytoma variant translocation 1 (PVT1) is involved in colorectal cancer (CRC), however, the underlying mechanism remains to be explored deeply, especially by in vivo models.

\section{Methods}

The expression level of PVT1 in CRC tissues was analyzed in TCGA and GEO database. The prognosis of PVT1 in CRC was assessed by analyzing the correlation between the expression level of PVT1 and overall survival in CRC patients by Kaplan-Meier survival analysis. The CCK-8 and colony formation assays were performed to study the proliferation of CRC cells, and transwell assays was used to assess the migration of CRC cells in vitro. The targeting relationships among PVT1, miR-24-3p and Neuropilin 1 (NRP1) was predicted by StarBase, and examined by dual-luciferase reporter assays. The expression levels of PVT1, miR-24-3p and NRP1 were quantified by quantitative real time PCR and/or western blotting. The zebrafish CRC xenograft models were used to study the function of PVT1, miR-24-3p and NRP1 in vivo.

\section{Results}

Bioinformatics analysis showed that PVT1 was upregulated in CRC tissues and highly associated with poor prognosis. Knockdown of PVT1 inhibited cell proliferation and migration of CRC cells, while overexpression of PVT1 promoted the progression of CRC cells in vitro. In zebrafish xenografts, silencing of PVT1 also suppressed the growth and metastasis of CRC cells. miR-24-3p, binding with PVT1, negatively regulated the progression of CRC cells. Inhibition of miR-24-3p counteracted the effects of PVT1 knockdown. In addition, miR-24-3p could regulate CRC progression by targeting NRP1 directly. Moreover, the expression of NRP1 was regulated by PVT1, and NRP1 overexpression could partially rescue the inhibition effects of PVT1 knockdown on CRC progression in vitro and in vivo.

\section{Conclusions}

Our study reveals that PVT1 promoted the proliferation and metastasis of CRC via regulating miR-24$3 p / N R P 1$ axis, and it could be a prognosis biomarker of CRC. This study provides a potential therapeutic target for CRC patients.

\section{Background}

Colorectal cancer (CRC) is one of common malignant cancers with high morbidity and mortality, it is the second cause of cancer death in the world [1,2]. In recent years, although early diagnosis and new therapeutic approaches for CRC patients are developed rapidly, their poor prognosis and overall survival (OS) rate have not been improved significantly [3-5]. Therefore, understanding the molecular mechanisms in the development and progression of CRC is critical for changing such situations. 
Long non-coding RNAs (IncRNAs), a class of non-coding transcripts more than 200 nucleotides in length, have been thought to be transcriptional "noise" before [6-8]. In recent years, it is proved that IncRNAs can regulate gene expression both at transcriptional and post-transcriptional levels [9, 10]. Many IncRNAs also have been identified to play important roles in human diseases, especially in cancer [11-16]. It is reported that IncRNAs regulate tumor proliferation, apoptosis, migration and invasion, some of them have been identified as a potential prognostic biomarker of cancer [17-19]. The IncRNA plasmacytoma variant translocation 1 (PVT1) is located on human chromosome 8q24, which is the star genomic region for human cancer studies. The oncogene c-Myc locates in this region [20, 21], and it has been verified which plays oncogenic roles in various human cancers, including lung cancer, breast cancer, and colorectal cancer [22]. Similar to c-Myc, PVT1 is also involved in multiple tumors and correlated with poor prognosis $[21,23,24]$. However, the precise molecular mechanism of PVT1 in CRC needs to investigate furtherly.

Many of IncRNAs, including PVT1, act as competing endogenous RNAs (ceRNAs) which competitively absorb miRNAs and indirectly regulate the expression of miRNA targeting genes [25-27]. It has been reported PVT1 can participate the progression of CRC via ceRNA mechanisms, such as PVT1/miR455/RUNX2 axis, PVT1/miR-106b-5p/FJX1 axis, PVT1/miR-216a-5p/YBX1 axis [28]. PVT1 also can act as a sponge of miR-24-3p in LPS-induced cardiac fibroblasts and ischemic stroke [29, 30], while such regulation in tumor progression is still unclear.

Due to the difficulty, most of ceRNA network studies have limited in vivo experiments, especially for tumor metastasis. Zebrafish xenograft model has been developed as a rapid and promising model for human cancers in recent years [31-33]. So far, more and more zebrafish cell line derived xenograft (zCDX) models have been established successfully, such as colorectal cancer, breast cancer, prostate cancer, lung cancer [32,34], and they also have been applied to study the roles of IncRNA in human cancers [35]. Compared to mouse xenograft models, zebrafish xenografts can be used to evaluate the proliferation and metastasis of tumor cells simultaneously by direct observation in vivo [32, 36-38]. Taking these advantages, zebrafish xenograft provides an easy in vivo animal model for studying ceRNA network.

In this study, we found that PVT1 was upregulated in CRC tissues and correlated with poor prognosis by analyzing the public database of patients. Functional experiments showed that PVT1 promoted the proliferation and metastasis of CRC cells in vitro and in vivo. Furthermore, molecular mechanism studies showed that PVT1 up-regulated the expression of NRP1 through competitively sponging to miR-24-3p, and inhibition of miR-24-3p or overexpression of NRP1 could rescued the suppression effects of PVT1 silence partially in CRC cells.

\section{Methods}

\section{TCGA and GEO database analysis}

The expression levels of IncRNA PVT1 in tumor tissue samples was analyzed through Gene Expression Profiling Interactive Analysis (GEPIA) (http://gepia.cancer-pku.cn/) using The Cancer Genome Atlas 
(TCGA) database, which contains 275 colon adenocarcinoma tissue samples and 41 normal tissue samples, 92 rectum adenocarcinoma tissue samples and 10 normal tissue samples. The dataset GSE18105 from Gene Expression Omnibus (GEO) database was used to analyze the expression levels of PVT1 in CRC tissues by InCAR software (https://lncar.renlab.org/), which contains 94 tumor samples and 17 normal samples. And the dataset GSE41258 was used to compare the expression of PVT1 in primary and metastasis CRC tissues, which includes 67 metastasis tumors and 186 primary tumors samples. The OS of CRC patients was analyzed by Kaplan-Meier method (https://kmplot.com/analysis/).

\section{Cell Culture}

The human CRC cell lines were obtained from Institute of Biochemistry and Cell Biology of Chinese Academy of Science (Shanghai, China). HCT116, SW620 and FHC cells were cultured in 1640 medium, SW480 cells were cultured in DMEM medium, and LoVo cells were cultured in F-12K medium. All culture media were supplemented with $10 \%$ fetal bovine serum (FBS), and all cells were cultured in a humidified atmosphere with $5 \%$ at $37^{\circ} \mathrm{C}$.

\section{Rna Exaction And Quantitative Real-time Pcr}

Total RNA was exacted from cultured cells using TRIzol reagent (Invitrogen, CA, USA). Then the total RNA was reverse transcribed to CDNA using random primers according to instructions of PrimeScript RT kit (Takara, Dalian, China). Then, the expression levels of different genes were detected by quantitative realtime PCR (qRT-PCR) using SYBR Green Master Mix kit (Takara, Dalian, China). And glyceraldehyde 3phosphate dehydrogenase (GAPDH) was used as endogenous control. The specific primers were used for reverse transcription of miRNA, and the sequences were: $5^{\prime}-$ GTTGGCTCTGGTGCAGGGTCCGAGGTATTCGCACCAGAGCCAACCTGTTC-3' (miR-24-3p), 5'GTTGGCTCTGGTGCAGGGTCCGAGGTATTCGCACCAGAGCCAACCGATGT-3' (miR-455-5p), 5'GTTGGCTCTGGTGCAGGGTCCGAGGTATTCGCACCAGAGCCAACAGGAGA-3' (miR-605-5p), 5'GTTGGCTCTGGTGCAGGGTCCGAGGTATTCGCACCAGAGCCAACGAGCTA-3' (miR-143-3p), 5'GTTGGCTCTGGTGCAGGGTCCGAGGTATTCGCACCAGAGCCAACACAAAC-3'(miR-497-5p). U6 was used as internal control for detecting miRNA expression. All data were analyzed by $2^{-\Delta \Delta C t}$ methods. The primer sequences for qRT-PCR were listed in Table 1. 
Table 1

Primer sequences for qRT-PCR

\begin{tabular}{|c|c|c|}
\hline Gene & Forward primer $\left(5^{\prime}-3^{\prime}\right)$ & Reverse primer $\left(5^{\prime}-3^{\prime}\right)$ \\
\hline PVT1 & TTGGCACATACAGCCATCAT & CAGTAAAAGGGGAACACCA \\
\hline NRP1 & cgggacccattcaggatcac & gctgatcgtactcctctggc \\
\hline GAPDH & GGGAGCCAAAAGGGTCAT & GAGTCCTTCCACGATACCAA \\
\hline miR-24-3p & GTTTGGCTCAGTTCAGCAG & GTGCAGGGTCCGAGGT \\
\hline miR-455-5p & GGGTATGTGCCTTTGGACT & GTGCAGGGTCCGAGGT \\
\hline miR-605-5p & GGTAAATCCCATGGTGCCT & GTGCAGGGTCCGAGGT \\
\hline miR-143-3p & GTGGTGAGATGAAGCACTG & GTGCAGGGTCCGAGGT \\
\hline miR-497-5p & GTTTCAGCAGCACACTGTG & GTGCAGGGTCCGAGGT \\
\hline U6 & GCTTCGGCAGCACATATACTAAAAT & CGCTTCACGAATTTGCGTGTCAT \\
\hline
\end{tabular}

\section{Cell Transfection}

A small interfering RNA (siRNA) targeting PVT1 (si-PVT1) (5'-cagccatcatgatggtact-3'), negative control (NC) siRNA (5'-TTCTCCGAACGTGTCACGT-3'), miR-24-3p mimics (5'-TGGCTCAGTTCAGCAGGAACAg-3') or inhibitor (5'-CTGTTCCTGCTGAACTGAGCCA-3') were all purchased from General Biosystems (China). The control plasmid (pcDNA3.1), overexpression plasmids (pcDNA3.1-PVT1 or pcDNA3.1-NRP1) were synthesized by General Biosystems (China). The cells were cultured into six-well plates, and the siRNAs or plasmids were transfected into the cells using lipofectamine 2000 reagents (Invitrogen, USA) according to the manufacturer's protocol. After $24 \mathrm{~h}$, the silencing efficiencies were examined by qRT-PCR.

\section{Cck-8 Assay}

Cell proliferation was evaluated using Cell Counting Kit-8 (CCaK-8, DOJINDO, Japan). The transfected cells were seeded at a density of 2000 cells /well in 96-well plates. $10 \mu \mathrm{L}$ CCK-8 reagent containing 100 $\mu \mathrm{L}$ medium was added into each well, and then cultured for $2 \mathrm{~h}$. Cell proliferation was quantified by measuring the optional density at $450 \mathrm{~nm}$ on a microplate reader (BioTek Elx800, USA) every 24 hours from 0 to 96 hours according to the manufacturer's instructions.

\section{Colony Formation Assay}

The transfected cells were seeded into six-well plates with a density of 600 cells / well. The culture media, containing $10 \%$ FBS, $100 \mathrm{U} / \mathrm{ml}$ penicillin and $100 \mu \mathrm{g} / \mathrm{ml}$ streptomycin, were replaced every three days. 
After 2 weeks, the colonies were washed twice with PBS, fixed with methanol for 10 min and then stained with $0.1 \%$ crystal violet for $10 \mathrm{~min}$. The stained colonies were photographed and counted.

\section{Transwell Assay}

Cell migration was evaluated by transwell assay using 24-well plates with $8 \mu \mathrm{m}$ pore size chamber inserts. The transfected cells of $5 \times 10^{4}$ diluted with $200 \mu \mathrm{L}$ serum-free medium were seeded into the upper chambers of transwell plates. Then the upper chambers were placed into the lower chambers in 24-well plates containing $800 \mu \mathrm{L}$ medium with $10 \%$ FBS. After $24 \mathrm{~h}$, the cells were fixed using methanol for 10 min and stained using $0.1 \%$ crystal violet for $10 \mathrm{~min}$. Then the cells were imaged under the inverted microscope (10X).

\section{Western Blot}

The transfected cells were lysed by Radio Immunoprecipitation Assay Lysis buffer (RIPA, Beyotime, China) for total protein extraction. The protein samples were separated by sodium dodecyl sulfatepolyacrylamide gel electrophoresis and electro-transferred on polyvinylidene fluoride membranes (Millipore, Schwalbach, Germany), then were blocked with 5\% skim milk. Subsequently, the membranes were incubated with primary antibody (BOSTER, China) which for neuropilin-1 (1:500) or GAPDH (1:10000) at $4{ }^{\circ} \mathrm{C}$ overnight. Then the membranes were put into the secondary antibody (1:5000, BOSTER, China) for 1 hours at room temperature. After washing, the proteins were visualized with a BeyoECL plus kit (Beyotime, China).

\section{Luciferase Reporter Assay}

The potential binding site of PVT1 or NRP1 3' UTR with miR-24-3p was predicted by StarBase (https://starbase.sysu.edu.cn/), miRDB (http://mirdb.org/) and DIANA (http://diana.imis.athenainnovation.gr/). The wild-type and mutant-type fragments of PVT1 and NRP1 3' UTR were synthesized and cloned into pGL3-basic vector. The reporter plasmids were co-transfected with miR-24-3p mimics or NC mimics into HEK-293T cells using Lipofectamine 2000 reagents. After $48 \mathrm{~h}$, the luciferase activity was detected by a dual-luciferase assay kit (Promega, UAS) according to the manufacturer's protocols.

\section{Zebrafish Xenograft Model}

The adult $\mathrm{Tg}$ (fli1a:EGFP) zebrafish were maintained at $28{ }^{\circ} \mathrm{C}$ and $14 \mathrm{~h}-10 \mathrm{~h}$ light-dark cycle in a fish auto culture system (Haishen, China). The embryos of zebrafish were cultured into $10 \%$ Hank's solution, which was composed of (in $\mathrm{mM}$ ) $140 \mathrm{NaCl}, 5.4 \mathrm{KCl}, 0.25 \mathrm{Na}_{2} \mathrm{HPO}_{4}, 0.44 \mathrm{KH}_{2} \mathrm{PO}_{4}, 1.3 \mathrm{CaCl}_{2}, 1.0 \mathrm{MgSO}_{4}$ and 4.2 $\mathrm{NaHCO}_{3}$ (pH 7.2). The 48-hpf (hours post-fertilization) Tg(fli1a:EGFP) zebrafish embryos were used for 
cancer cell transplant experiments in our study [39]. Zebrafish handling procedures were approved by Lianshui County People's Hospital, Kangda College of Nanjing Medical University.

Before injection, the tumor cells, transfected with siRNA or/and plasmid, were harvested and stained by CM-Dil (Invitrogen, USA) [35]. The 48-hpf zebrafish larvae were mounted with 1.2\% low-melting agarose (Promega, USA), then approximately 300-400 labeled cells were injected into the perivitelline space (PVS) of zebrafish larvae under a microinjector (Picosprizer III, USA). Then the injected zebrafish larvae were cultured at $34{ }^{\circ} \mathrm{C}$ in light incubator. At 1 day post injection (dpi), the larvae with similar sizes of fluorescence area were selected for further research and cultured at $34{ }^{\circ} \mathrm{C}$ until the end of experiments. At $4 \mathrm{dpi}$, the zebrafish larvae were mounted with $1.2 \%$ low-melting agarose, then the images were acquired by stereotype microscope (MVX10, Olympus, Japan) or confocal microscope using 20X objective lens (Fluoview 3000, Olympus, Japan). The resolution of the images was $1600 \times 1200$ (MVX10) or 1024×1024 pixels (Fluoview 3000).

\section{Statistical analysis}

All the statistical data were analyzed using unpaired Student's t-test and their figures were generated by GraphPad Prism 7.0. The values of $P<0.05$ were considered to be statistically significant. All results were presented as the mean \pm SD.

\section{Results}

\section{PVT1 is upregulated in CRC tissues and associated with poor prognosis of CRC patients}

Firstly by analyzing TCGA database, we found the expression of PVT1 was significantly upregulated both in colon adenocarcinoma (COAD) and rectum adenocarcinoma (READ) comparing to the normal tissues (Fig. 1A, P < 0.05). The data of GEO dataset GSE18105 also showed that the expression of PVT1 was upregulated in CRC tissues (Fig. 1B, P < 0.001). To study the relationship between PVT1 and the prognosis of CRC, we then analyzed another GEO dataset GSE41258 and found the expression of PVT1 was much higher in metastasis tumors than that in primary tumors of CRC patients (Fig. 1C, $P<0.001$ ). Furthermore, the OS of CRC patients was also analyzed and showed high PVT1 expression level was correlated with low survival rate of CRC patients (Fig. 1D, $P<0.05$ ). These results present that the expression of PVT1 is upregulated in CRC tissues, and also associated with poor prognosis of CRC patients.

\section{PVT1 promotes the proliferation and migration of CRC cells in vitro}

We examined the expression levels of PVT1 in four CRC cell lines (HCT116, SW480, SW620 and LoVo) and human normal colon epithelial cell (FHC). Compared with FHC, and found that PVT1 was highly expressed in CRC cells, especially in HCT116 and SW480 cell lines (Fig. 2A, P < 0.05). To verify the 
biological functions of PVT1 in CRC cells, we transfected small interference RNA (siRNA) or overexpression plasmid to downregulate or upregulate the expression of PVT1 in CRC cells. Compared with negative control siRNA (NC), PVT1 siRNA (si-PVT1) transfection efficiently decreased the expression of PVT1, which the knockdown efficiency was $79.7 \%$ and $89.4 \%$ in HCT116 and SW480 cells, respectively (Fig. 2B, $\mathrm{P}<0.01$ ). Overexpression plasmid (pcDNA3.1-PVT1) transfection increased the expression level of PVT1 by 128 times compared to control (pcDNA3.1 empty vector transfection) (Fig. $2 \mathrm{C}, \mathrm{P}<0.001$ ). Then we used CCK-8 to assess the proliferation of CRC cells, and found PVT1 knockdown inhibited the proliferation of HCT116 (Fig. 2D, P < 0.001) and SW480 cells (Fig. 2E, P < 0.001), but overexpression PVT1 increased the proliferation of LoVo cells (Fig. 2F, P < 0.001). And colony formation assays also verified the proliferation role of PVT in CRC (Fig. 2G-I, P < 0.05). Subsequently, we used transwell assays to assess the migration of CRC cells, and found silence of PVT1 significantly suppressed the cell migration in HCT116 (Fig. 2J, P < 0.01) and SW480 cells (Fig. 2L, P < 0.05). On the contrary, the migration of LoVo cells was enhanced when overexpressing PVT1 (Fig. 2L, P < 0.05). Taken together, these data shows that PVT1 promotes the proliferation and migration of CRC cells in vitro.

\section{Knockdown of PVT1 inhibits the growth and metastasis of CRC cells in vivo}

To verify the functional roles of PVT1 in CRC cells in vivo, the zebrafish xenograft models were used. HCT116, transfected with si-PVT1 or NC and labeled by CM-Dil, were transplanted into the PVS of 48-hpf (hours post fertilization) $\mathrm{Tg}$ (fli1a: EGFP) transgenic zebrafish larvae, which the vascular endothelial cells were labeled by EGFP. At 4 days post injection (dpi), we quantified the area of CM-Dil positive signals in yolk representing the cell growth (Fig. 3A, B), and found silencing PVT1 inhibited the growth of HCT116 compared with NC groups (Fig. 3C, P<0.05). We then quantified the area of CM-Dil positive in trunk (Fig. 3D, E) representing cell metastasis, and found PVT1 knockdown also inhibited the metastasis of HCT 116 cells (Fig. 3F, P < 0.05). Similarly, knocking-down PVT1 also inhibited the growth and metastasis of SW480 cells (Fig. 3G-L, P< 0.05 ). These results demonstrate that PVT1 regulates the growth and metastasis of CRC cells in vivo.

\section{PVT1 negatively regulates the expression of miR-24-3p as a sponge in CRC cells}

Previous studies have revealed that PVT1 can act as a miRNA sponge to regulate the progression of various tumors $[22,40]$. We used three online tools to predict candidate miRNAs which could bind with PVT1, including StarBase, miRDB and DIANA Tools. By cross comparison, we found miR-24-3p, miR-1433p, miR-455-5p, miR-497-5p and miR-605-5p were predicted by all the three online tools (Fig. 4A). Then we examined the expressions of these miRNAs when knocking-down PVT1, and screened out three miRNAs (miR-24-3p, miR-455-5p, miR-605-5p) whose expression levels were upregulated when PVT1 silence in SW480 cells (Fig. 4B, $\mathrm{P}<0.05$ ). It is reported that miR-24-3p plays regulation roles in proliferation, migration and invasion of CRC cells [41], and our analysis also showed its potential association with the poor prognosis of CRC (Fig. 4C). Therefore, we chose miR-24-3p for the further studies. According to the predicted binding site between PVT1 and miR-24-3p by StarBase (Fig. 4D), dual luciferase reporter assays were performed for the verification in vitro. Compared with control, we found luciferase activity was suppressed when co-transfecting PVT1 reporter plasmid and miR-24-3p mimics in HEK-293T cells 
(Fig. 4E, $\mathrm{P}<0.01$ ), while such regulation was totally blocked when co-transfecting the mutant PVT1 reporter plasmid and miR-24-3p mimics (Fig. 4D, E). These results suggest that PVT1 negatively regulate the expression of miR-24-3p through binding with miR-24-3p directly in CRC cells.

Knockdown of miR-24-3p promotes the proliferation and metastasis of CRC cells in vitro and in vivo

To study the roles of miR-24-3p in CRC, we efficiently silenced the expression of miR-24-3p by transfecting miR-24-3p inhibitor (Fig. 4F, P< 0.001). CCK-8 and transwell assays showed that miR-24-3p inhibition promoted the proliferation and migration of SW480 cells in vitro (Fig. 4G, H). By using zebrafish xenograft models, knockdown of miR-24-3p also promoted the growth and metastasis of CRC cells in vivo (Fig. $4 \mathrm{I}, \mathrm{J}$ ). These data show that miR-24-3p negatively regulates the progression of CRC cells in vitro and in vivo.

\section{Knockdown Of Mir-24-3p Rescues The Progression Of Crc Cells Which Repressed By Pvt1 Silence}

To determine whether miR-24-3p mediated the regulation of PVT1 in CRC progression, we studied it by cotransfecting si-PVT1 and miR-24-3p inhibitor into SW480 cells simultaneously. Similar to our previous results, knockdown of PVT1 suppressed the proliferation of CRC cells, but miR-24-3p inhibition counteracted the suppression effects which caused by PVT1 knockdown (Fig. 5A, P < 0.05). Transwell assay also showed that silence of miR-24-3p partly restored the ability of cell migration of CRC cells which suppressed by PVT1 knockdown (Fig. 6B, P<0.05). These results indicate that PVT1 promotes the progression of $C R C$ cells via regulating the expression of miR-24-3p.

\section{Nrp1 Is Directly Target Of Mir-24-3p In Crc Cells}

To find the direct downstream target genes of miR-23-3p, we also used StarBase, miRDB and DIANA tools to predict. A serious of tumor-related genes were predicted by cross comparison, such as NRP1, PAK4, MMP14, KLF4, FSCN1 (Fig. 6A). We selected NRP1 for further research because it was reported that NRP1 involved in the progression of CRC [42]. Then we overexpressed miR-24-3p by transfecting its mimics into SW480 cells, and found the expression of NRP1 were decreased both in transcriptional and translational expression level (Fig. 6B, C, P < 0.01). According to the binding site between $3^{\prime} U T R$ of NRP1 and miR-24$3 p$ which was also predicted by StarBase tool (Fig. 6D), we did the dual luciferase reporter assays and found miR-24-3p could bind with 3'UTR of NRP1 directly (Fig. 6E, P $<0.001$ ). These data show that miR24-3p can downregulate the expression of NRP1 in CRC cells by binding the 3'UTR of NRP1 directly.

\section{Overexpression Nrp1 Rescues The Progression Of Crc Cells Which Suppressed By Mir-24-3p Overexpression}


To determine whether NRP1 mediated the regulation of miR-24-3p in CRC progression, we tried to perform similar experiments by cotransfecting NRP1-overexpressing plasmid (pcDNA3.1-NRP1) and miR-24-3p mimic into SW480 cells simultaneously. First, we transfected pcDNA3.1-NRP1 into SW480 cells and examined the overexpression efficiency by qRT-PCR and western blot. We found NRP1-overexpressing plasmid transfection could efficiently upregulated the expression level of NRP1 (Fig. 6F, G, P< 0.01). NRP1 overexpression not only promoted the proliferation of CRC cells, but also rescued the CRC proliferation which suppressed by miR-24-3p overexpression (Fig. $6 \mathrm{H}, \mathrm{P}<0.05$ ). The data of transwell assays also showed the similar rescue roles when overexpressing NRP1 (Fig. $61, P<0.05$ ). These results suggest miR-24-3p regulates the progression of CRC cells via targeting NRP1.

\section{Knockdown of PVT1 suppressed the growth and metastasis of CRC cells by regulating NRP1 expression} in vitro and in vivo

To investigate whether PVT1 functioned through NRP1 pathway in CRC cells, we firstly detected the expression level of NRP1 when knocking-down PVT1 in SW480 cells. We found PVT1 knockdown reduced the expression level of NRP1 (Fig. 7A, B, P < 0.001). Then we cotransfected si-PVT1 and pcDNA3.1-NRP1 in CRC cells, and we also found the suppression effects which caused by PVT1 knockdown were partially counteracted by NRP1 overexpression both in proliferation and migration levels (Fig. 7C, D, P < 0.01). NRP1-related OS analysis in CRC patients indicated its clinical significance (Fig. 7E, $\mathrm{P}<0.01$ ), and we then examined the PVT1/NRP1 regulatory pathway in zebrafish xenografts. In vivo experiments also showed that NRP1 overexpression could rescue the CRC progression which suppressed by PVT1 knockdown (Fig. 7F, G, P< 0.05). The results indicate that PVT1 regulates the progression of CRC cells through NRP1 pathway.

\section{Discussion}

It is reported that PVT1 is elevated in multiple cancers, such as gastric cancer, breast cancer, lung cancer [43]. PVT1 has be identified which plays oncogenic roles through various pathways and is associated with poor prognosis of CRC patients [23]. In the present study, we found that PVT1 was upregulated in CRC tissues and predicted the poor prognosis by analyzing public database. Functional studies demonstrated that PVT1 could promote the proliferation and metastasis of CRC cells in vitro and in vivo. These results verify the oncogenic roles of PVT1 in CRC.

PVT1 has been identified that it can function as miRNA sponges to regulate miRNA-targeting genes in CRC cells $[22,26,30,43]$. In this study, we screened the miRNAs which could be absorbed by PVT1 in CRC cells. And we found miR-24-3p could bind with PVT1 directly, and its expression level was regulated by PVT1. It is also reported that miR-24-3p functions as a tumor suppressor, which is downregulated in CRC tissues and inhibits the proliferation, migration and invasion of CRC cells [41]. In previous study, PVT1 has been proved that can sponge miR-24-3p in LPS-induced cardiac fibroblasts and ischemic stroke, while thus regulation has not been proved in cancers. In this study, bioinformatics analysis indicated the expression level of miR-24-3p and OS of CRC patients were negatively correlated. Moreover, we also 
found miR-24-3p knockdown not only promoted the progression of $C R C$, but also prevented the inhibition effects of PVT1 silence. These data demonstrate that PVT1 can functions as an oncogene by sponging miR-24-3p in CRC.

NRP1 was proved to promote the cell viability, migration, and invasion in CRC [42], and it was also a target gene of miR-24-3p 7 [44, 45]. Bioinformatics analysis indicated that the expression level of NRP1 predicted the poor prognosis of CRC (Fig. 7E). Based of it, we furtherly studied whether miR-24-3p/NRP1 axis played roles in the progression of CRC cells. Our data showed miR-24-3p could directly bind with the 3'UTR of NRP1 and regulate the proliferation and migration of CRC cells through NRP1. Then we investigated whether PVT1 functioned through NRP1 in CRC. And we also found PVT1 knockdown decreased the expression of NRP1, NRP1 overexpression could rescued the tumor progression which inhibited by PVT1 silence in CRC cells both in vitro and in vivo. These results suggest PVT1 can function as a ceRNA by regulating miR-24-3p/NRP1 axis in CRC cells.

Mouse xenograft models are widely applied for human cancer research, which is also used for verification of PVT1 in the proliferation of CRC, however the metastasis mouse model for studying the function of PVT1 is very few $[46,47]$. Compared with mouse models, zebrafish xenografts have multiple advantages in tumor biology. First, we can access the proliferation and metastasis of tumor cells in 96 hours after transplantation. Second, the proliferation and metastasis can be analyzed simultaneously by monitoring the behavior of tumor cell in vivo due to the transparent zebrafish larvae. Third, the genetic operation was relatively easy. Instead of shRNA plasmid transfection, siRNA transfected tumor cells can be used for the verification in 4 days. Using mouse models, He and his colleagues reported PVT1 overexpression promoted the metastasis of CRC cells from spleen parenchyma to liver during 28 days post-injection [46]. In this study, we showed that silencing PVT1 by siRNA transfection also inhibited the proliferation and metastasis of CRC cells at 4 dpi in zebrafish xenografts, which was consisted with that in mouse models. By taking advantage of zebrafish xenografts, we furtherly not only verified the relationship between miR-24-3p and NRP1 in CRC progression, but also demonstrated the regulatory mechanism between PVT1 and NRP1 in vivo. These data suggest that zebrafish xenograft could be a reliable and feasible model for human cancer research, especially for ceRNA mechanism verification in vivo.

\section{Conclusions}

In summary, our results demonstrated the oncogenic roles of PVT1 which also predicted the poor prognosis of CRC patients. Additionally, we revealed a novel signaling pathway of PVT1 in CRC, which functioned as a ceRNA by regulating miR-24-3p/NRP1 axis. Our results indicate PVT1 could be a molecular marker of diagnostic and prognosis, and also be a potential treatment target for CRC patients.

\section{Abbreviations}


LncRNA: long noncoding RNA; PVT1: plasmacytoma variant translocation 1; CRC: colorectal cancer; OS, overall survival; ceRNA: competing endogenous RNAs; COAD: colon adenocarcinoma; READ: rectum adenocarcinoma; TCGA: The Cancer Genome Atlas; GEPIA: Gene Expression Profiling Interactive Analysis; GEO: Gene Expression Omnibus; qRT-PCR: quantitative real-time PCR; CCK-8: Cell Counting Kit-8; RIPA: Radio Immunoprecipitation Assay Lysis buffer; hpf: hours post-fertilization; dpi: days post injection; PVS: perivitelline space; FBS: fetal bovine serum; GAPDH: glyceraldehyde 3-phosphate dehydrogenase; NC: negative control; NRP1: Neuropilin 1.

\section{Declarations}

\section{Acknowledgements}

Not applicable.

\section{Authors' contributions}

HY: Conceptualization; Data curation; Formal analysis; Visualization; SG: Data curation; Formal analysis; Visualization; Funding acquisition; Writing - original draft; GC: Investigation; Methodology; Resources; HY and XZ: Software; Resources; Validation;YZ: Supervision; Funding acquisition; Project administration; Validation; Writing - review \& editing; all authors have read and approved the manuscript.

\section{Funding}

This work was supported by the following organizations. The funding bodies played no role in the design of the study and collection, analysis, and interpretation of data and in writing the manuscript.

1. Research Fund of Lianshui County People's Hospital.

2. Natural Science Research Program of Huai'an Science and Technology Bureau,2021.

3. The Programof Innovation and Entrepreneurship Doctor of Jiangsu Province, 2021

\section{Availability of data and materials}

The datasets used and/or analyzed during the current study are available from the corresponding author on reasonable request.

\section{Ethics approval and consent to participate}

This study was performed in line with the principles of the Declaration of Helsinki. Approval was granted by the Ethics Committee of Lianshui County People's Hospital, Kangda College of Nanjing Medical University. All participants obtained written informed consent.

\section{Consent for publication}


Not applicable.

\section{Competing interests}

The authors declare that they have no competing interests.

\section{References}

1. Siegel RL, Miller KD, Fedewa SA, Ahnen DJ, Meester RGS, Barzi A, et al. Colorectal cancer statistics, 2017. CA Cancer J Clin. 2017;67(3):177-93.

2. Marmol I, Sanchez-de-Diego C, Pradilla Dieste A, Cerrada E, Rodriguez Yoldi MJ. Colorectal Carcinoma: A General Overview and Future Perspectives in Colorectal Cancer. Int J Mol Sci. 2017;18(1).

3. Lech G, Slotwinski R, Slodkowski M, Krasnodebski IW. Colorectal cancer tumour markers and biomarkers: Recent therapeutic advances. World J Gastroenterol. 2016;22(5):1745-55.

4. Phillips JD, Hasson RM. Surgical management of colorectal lung metastases. J Surg Oncol.2019;119(5):629-35.

5. Ciombor KK, Wu C, Goldberg RM. Recent therapeutic advances in the treatment of colorectal cancer. Annu Rev Med. 2015;66:83-95.

6. Kung JT, Colognori D, Lee JT. Long noncoding RNAs: past, present, and future. Genetics. 2013;193(3):651-69.

7. Nagano T, Fraser P. No-nonsense functions for long noncoding RNAs. Cell. 2011;145(2):178-81.

8. Ponting CP, Oliver PL, Reik W. Evolution and functions of long noncoding RNAs. Cell. 2009;136(4):629-41.

9. Zhang X, Wang W, Zhu W, Dong J, Cheng Y, Yin Z, et al. Mechanisms and Functions of Long NonCoding RNAs at Multiple Regulatory Levels. Int J Mol Sci. 2019;20(22).

10. Beermann J, Piccoli MT, Viereck J, Thum T. Non-coding RNAs in Development and Disease: Background, Mechanisms, and Therapeutic Approaches. Physiol Rev. 2016;96(4):1297-325.

11. Fang Y, Fullwood MJ. Roles, Functions, and Mechanisms of Long Non-coding RNAs in Cancer. Genomics Proteomics Bioinformatics. 2016;14(1):42-54.

12. Gutschner T, Diederichs S. The hallmarks of cancer: a long non-coding RNA point of view. RNA Biol. 2012;9(6):703-19.

13. Liu Y, Yang Y, Li L, Liu Y, Geng P, Li G, et al. LncRNA SNHG1 enhances cell proliferation, migration, and invasion in cervical cancer. Biochem Cell Biol. 2018:96(1):38-43.

14. Luo H, Xu C, Le W, Ge B, Wang T. IncRNA CASC11 promotes cancer cell proliferation in bladder cancer through miRNA-150. J Cell Biochem. 2019;120(8):13487-93.

15. Song W, Shi C. LncRNA RGMB-AS1 facilitates pancreatic cancer cell proliferation and migration but inhibits cell apoptosis via miR-574-3p/PIM3 axis. Am J Physiol Gastrointest Liver Physiol. 
2021;321(5):G477-88.

16. Sun M, Nie F, Wang Y, Zhang Z, Hou J, He D, et al. LncRNA HOXA11-AS Promotes Proliferation and Invasion of Gastric Cancer by Scaffolding the Chromatin Modification Factors PRC2, LSD1, and DNMT1. Cancer Res. 2016;76(21):6299-310.

17. Yu J, Han Z, Sun Z, Wang Y, Zheng M, Song C. LncRNA SLCO4A1-AS1 facilitates growth and metastasis of colorectal cancer through beta-catenin-dependent Wnt pathway. J Exp Clin Cancer Res. 2018;37(1):222.

18. Jiang L, Zhao XH, Mao YL, Wang JF, Zheng HJ, You QS. Long non-coding RNA RP11-468E2.5 curtails colorectal cancer cell proliferation and stimulates apoptosis via the JAK/STAT signaling pathway by targeting STAT5 and STAT6. J Exp Clin Cancer Res. 2019;38(1):465.

19. Zhu X, Tian X, Yu C, Shen C, Yan T, Hong J, et al. A long non-coding RNA signature to improve prognosis prediction of gastric cancer. Mol Cancer. 2016;15(1):60.

20. Tseng YY, Moriarity BS, Gong W, Akiyama R, Tiwari A, Kawakami H, et al. PVT1 dependence in cancer with MYC copy-number increase. Nature. 2014;512(7512):82-86.

21. Shigeyasu K, Toden S, Ozawa T, Matsuyama T, Nagasaka T, Ishikawa T, et al. The PVT1 IncRNA is a novel epigenetic enhancer of MYC, and a promising risk-stratification biomarker in colorectal cancer. Mol Cancer. 2020;19(1):155.

22. Liu X, Li L, Bai J, Li L, Fan J, Fu Z, et al. IncRNA PVT1 promotes progression of colorectal cancer by sponging miR-152-3p and regulating E2F3/MAPK8 signaling. Cancer Sci. 2021.

23. Fan $\mathrm{H}$, Zhu JH, Yao XQ. Long non-coding RNA PVT1 as a novel potential biomarker for predicting the prognosis of colorectal cancer. Int J Biol Markers. 2018;33(4):415-22.

24. Pan X, Li B, Fan N, Li J, Cai F, Zhao G, et al. Long Noncoding RNA PVT1 as a Potent Predictor of Prognosis in Cancers: a Meta-Analysis. Clin Lab. 2017;63(10):1657-66.

25. Salmena L, Poliseno L, Tay Y, Kats L, Pandolfi PP. A ceRNA hypothesis: the Rosetta Stone of a hidden RNA language? Cell. 2011;146(3):353-58.

26. Wang X, Cheng Z, Dai L, Jiang T, Li P, Jia L, et al. LncRNA PVT1 Facilitates Proliferation, Migration and Invasion of NSCLC Cells via miR-551b/FGFR1 Axis. Onco Targets Ther. 2021;14:3555-65.

27. Liang R, Tang Y. LINC00467 knockdown repressed cell proliferation but stimulated cell apoptosis in glioblastoma via miR-339-3p/IP6K2 axis. Cancer Biomark. 2020;28(2):169-80.

28. Zeng X, Liu Y, Zhu H, Chen D, Hu W. Downregulation of miR-216a-5p by long noncoding RNA PVT1 suppresses colorectal cancer progression via modulation of YBX1 expression. Cancer Manag Res. 2019;11:6981-93.

29. Chen Z, Fan T, Zhao X, Zhang Z. Depleting SOX2 improves ischemic stroke via IncRNA PVT1/microRNA-24-3p/STAT3 axis. Mol Med. 2021;27(1):107.

30. Dai Q, Hong Y, Li J. PVT1 knockdown inhibited the biological behavior of LPS-induced cardiac fibroblasts by regulating miR-24. Genes Genomics. 2021;43(9):1003-9. 
31. Snaar-Jagalska BE. ZF-CANCER: developing high-throughput bioassays for human cancers in zebrafish. Zebrafish. 2009;6(4):441-3.

32. Fior R, Povoa V, Mendes RV, Carvalho T, Gomes A, Figueiredo N, et al. Single-cell functional and chemosensitive profiling of combinatorial colorectal therapy in zebrafish xenografts. Proc Natl Acad Sci U S A. 2017;114(39):E8234-43.

33. Hason M, Bartunek P. Zebrafish Models of Cancer-New Insights on Modeling Human Cancer in a Non-Mammalian Vertebrate. Genes (Basel). 2019;10(11).

34. Teng Y, Xie X, Walker S, White DT, Mumm JS, Cowell JK. Evaluating human cancer cell metastasis in zebrafish. BMC Cancer. 2013;13:453.

35. Shen W, Pu J, Sun J, Tan B, Wang W, Wang L, et al. Zebrafish xenograft model of human lung cancer for studying the function of LINC00152 in cell proliferation and invasion. Cancer Cell Int. 2020;20:376.

36. Lee LM, Seftor EA, Bonde G, Cornell RA, Hendrix MJ. The fate of human malignant melanoma cells transplanted into zebrafish embryos: assessment of migration and cell division in the absence of tumor formation. Dev Dyn. 2005;233(4):1560-70.

37. Marques IJ, Weiss FU, Vlecken DH, Nitsche C, Bakkers J, Lagendijk AK, et al. Metastatic behaviour of primary human tumours in a zebrafish xenotransplantation model. BMC Cancer. 2009;9:128.

38. Osmani N, Goetz JG. Multiscale Imaging of Metastasis in Zebrafish. Trends Cancer. 2019;5(12):76678.

39. Lawson ND, Weinstein BM. In vivo imaging of embryonic vascular development using transgenic zebrafish. Dev Biol. 2002;248(2):307-18.

40. Wang H, Huang Y, Yang Y. LncRNA PVT1 Regulates TRPS1 Expression in Breast Cancer by Sponging miR-543. Cancer Manag Res. 2020;12:7993-8004.

41. Gao Y, Liu Y, Du L, Li J, Qu A, Zhang X, et al. Down-regulation of miR-24-3p in colorectal cancer is associated with malignant behavior. Med Oncol. 2015;32(1):362.

42. Chen ZP, Wei JC, Wang Q, Yang P, Li WL, He F, et al. Long noncoding RNA 00152 functions as a competing endogenous RNA to regulate NRP1 expression by sponging with miRNA206 in colorectal cancer. Int J Oncol. 2018;53(3):1227-36.

43. Wan L, Sun M, Liu GJ, Wei CC, Zhang EB, Kong R, et al. Long Noncoding RNA PVT1 Promotes NonSmall Cell Lung Cancer Cell Proliferation through Epigenetically Regulating LATS2 Expression. Mol Cancer Ther. 2016;15(5):1082-94.

44. Kuai F, Zhou L, Zhou J, Sun X, Dong W. Long non-coding RNA THRIL inhibits miRNA-24-3p to upregulate neuropilin-1 to aggravate cerebral ischemia-reperfusion injury through regulating the nuclear factor kappaB p65 signaling. Aging (Albany NY). 2021;13(6):9071-84.

45. Mone P, Gambardella J, Wang X, Jankauskas SS, Matarese A, Santulli G. miR-24 Targets the Transmembrane Glycoprotein Neuropilin-1 in Human Brain Microvascular Endothelial Cells. Noncoding RNA. 2021;7(1). 
46. He F, Song Z, Chen H, Chen Z, Yang P, Li W, et al. Long noncoding RNA PVT1-214 promotes proliferation and invasion of colorectal cancer by stabilizing Lin28 and interacting with miR-128. Oncogene. 2019;38(2):164-79.

47. Wu H, Wei M, Jiang X, Tan J, Xu W, Fan X, et al. IncRNA PVT1 Promotes Tumorigenesis of Colorectal Cancer by Stabilizing miR-16-5p and Interacting with the VEGFA/VEGFR1/AKT Axis. Mol Ther Nucleic Acids. 2020;20:438-50.

\section{Figures}

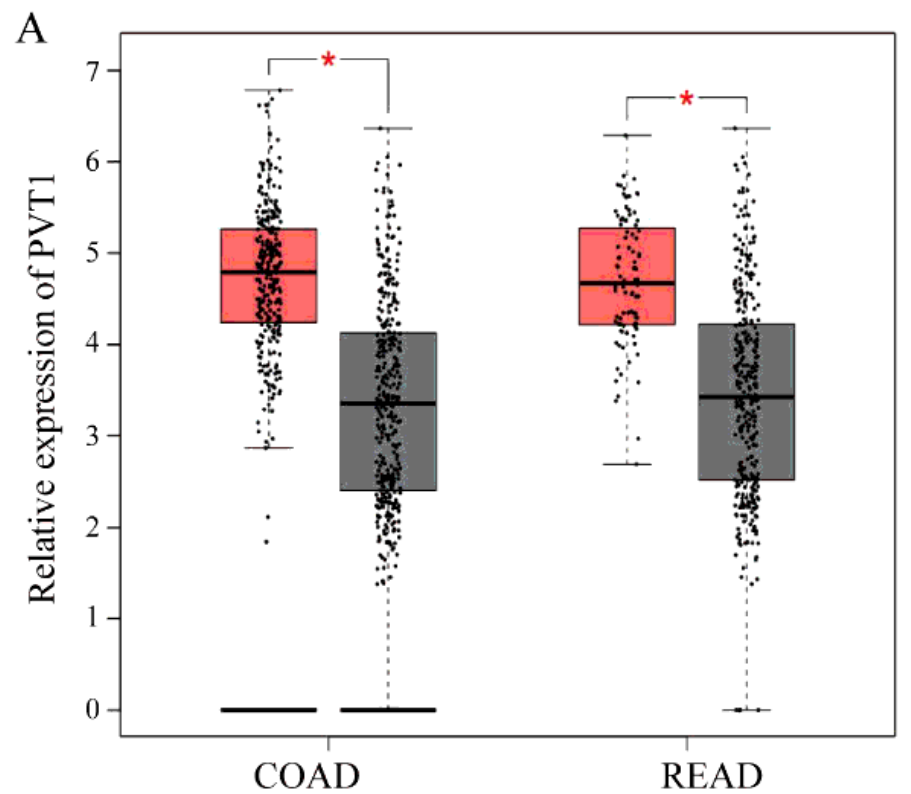

B

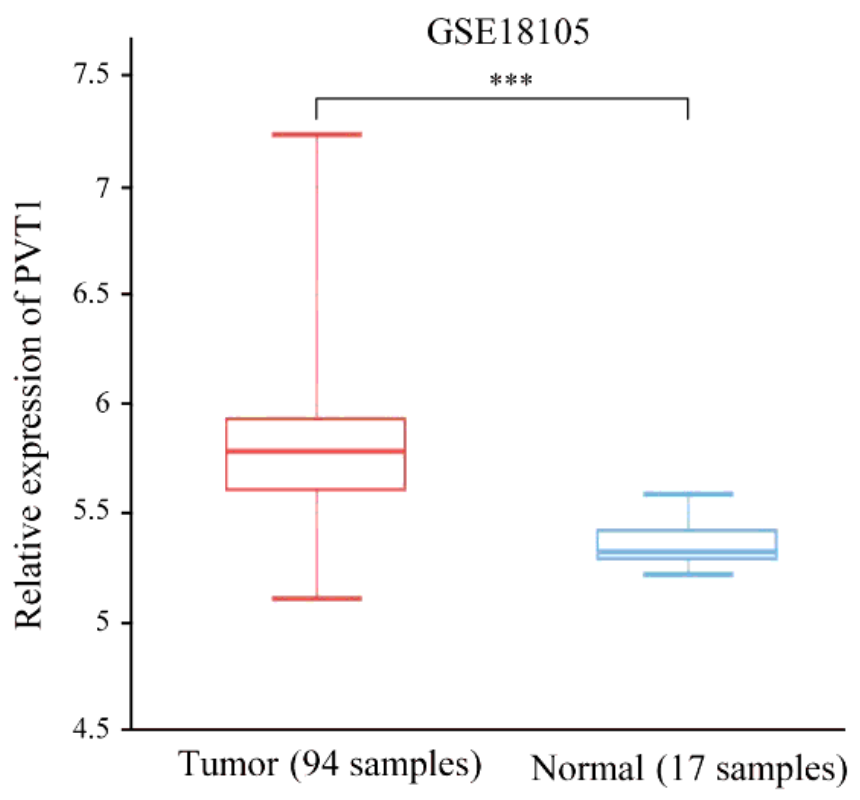

$(\operatorname{num}(\mathrm{T})=275 ; \operatorname{num}(\mathrm{N})=41) \quad(\operatorname{num}(\mathrm{T})=92 ; \operatorname{num}(\mathrm{N})=10)$

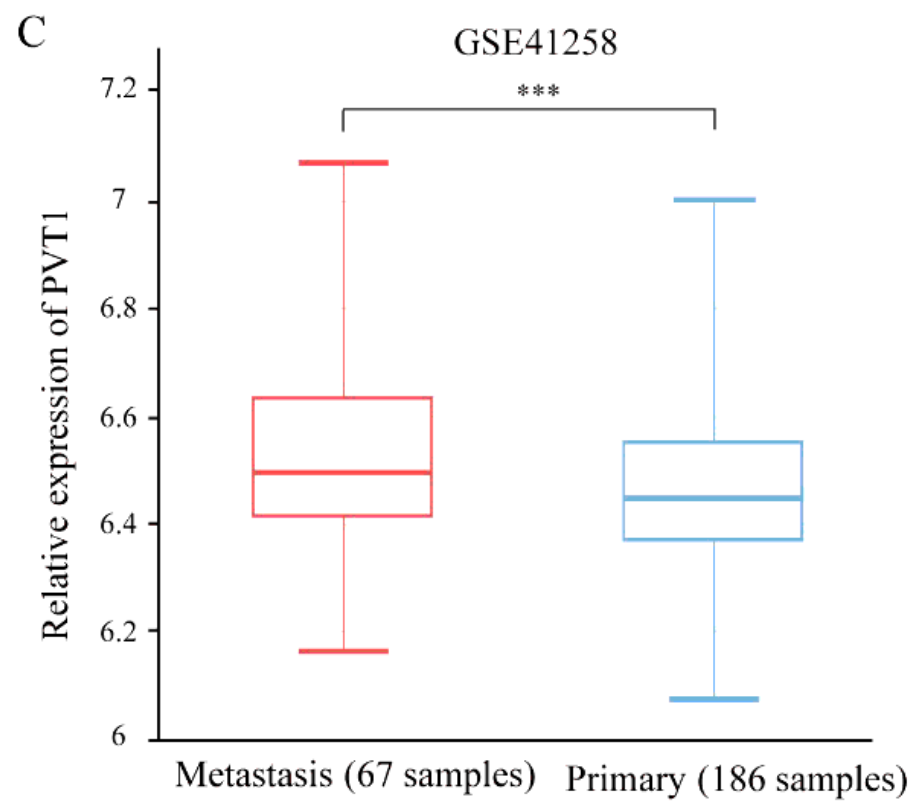

$\mathrm{D}$

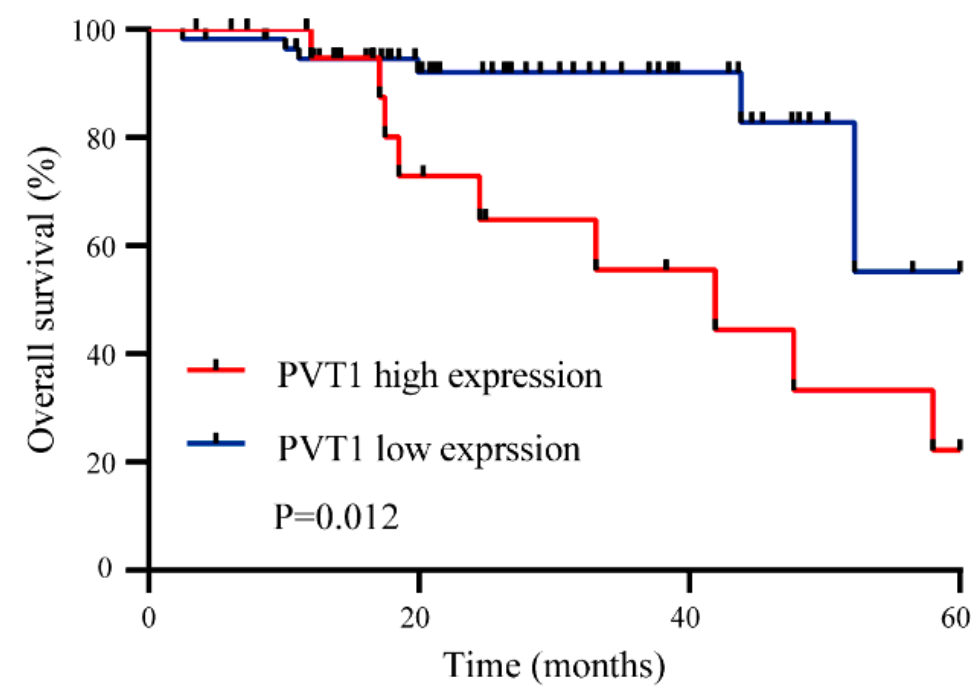

Figure 1 
PVT1 is upregulated in CRC tissues and associated with poor prognosis. A The expression of PVT1 in CRC tissues (COAD and READ, T) was analyzed byTCGA database comparing to normal tissues. $(\mathrm{N})$. B The expression of PVT1 in CRC tissues was analyzed by GEO dataset GSE18105 comparing to normal tissues. C The expression of PVT1 in metastasis CRC tissues was analyzed byGSE41258 comparing to primary CRC tissues. DOS of CRC patients with high PVT1 expression was analyzed by Kaplan-Meier analysis comparing to that with low PVT1 expression. *: $\mathrm{P}<0.05$, ***: $\mathrm{P}<0.001$.
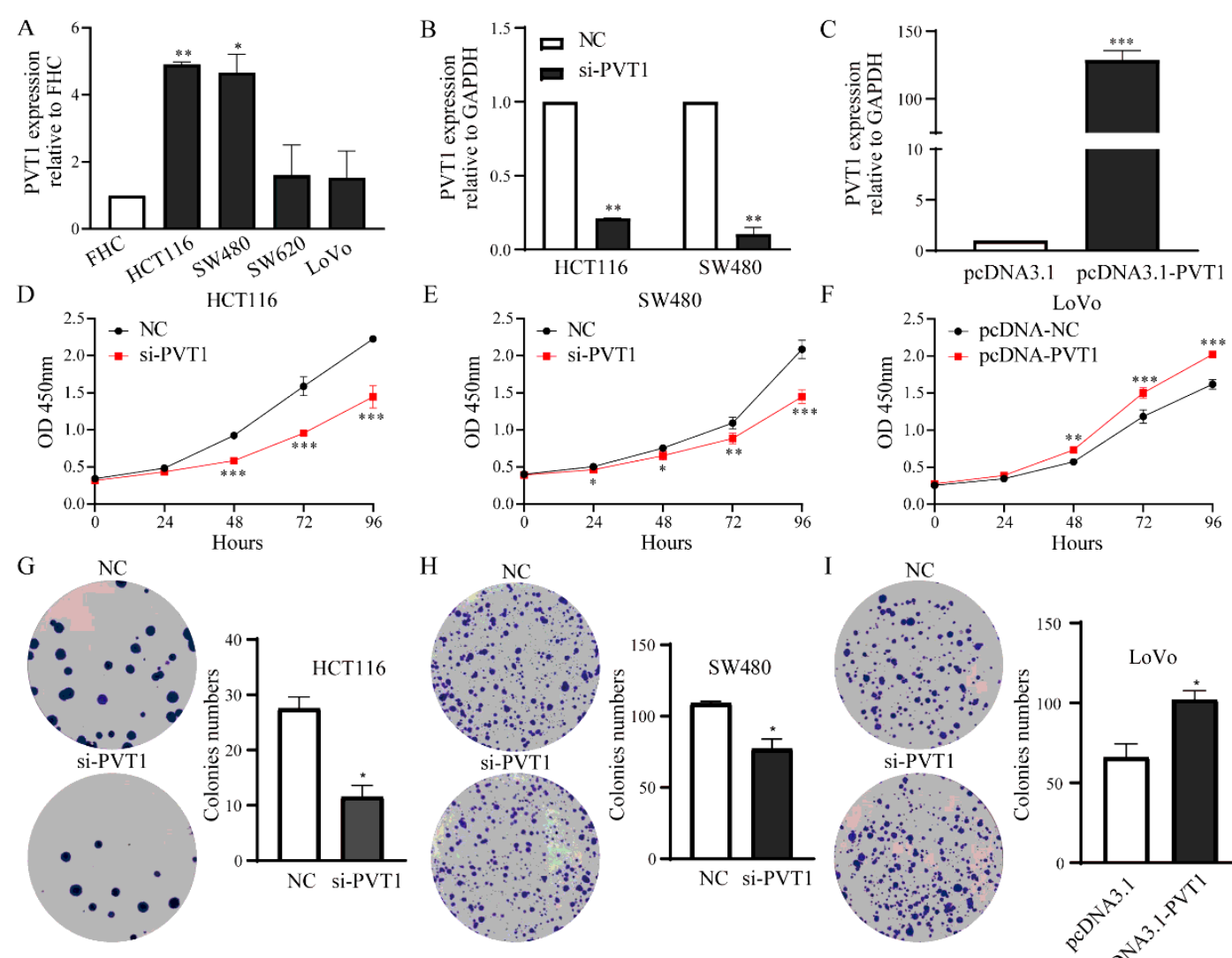

$\mathrm{J}$
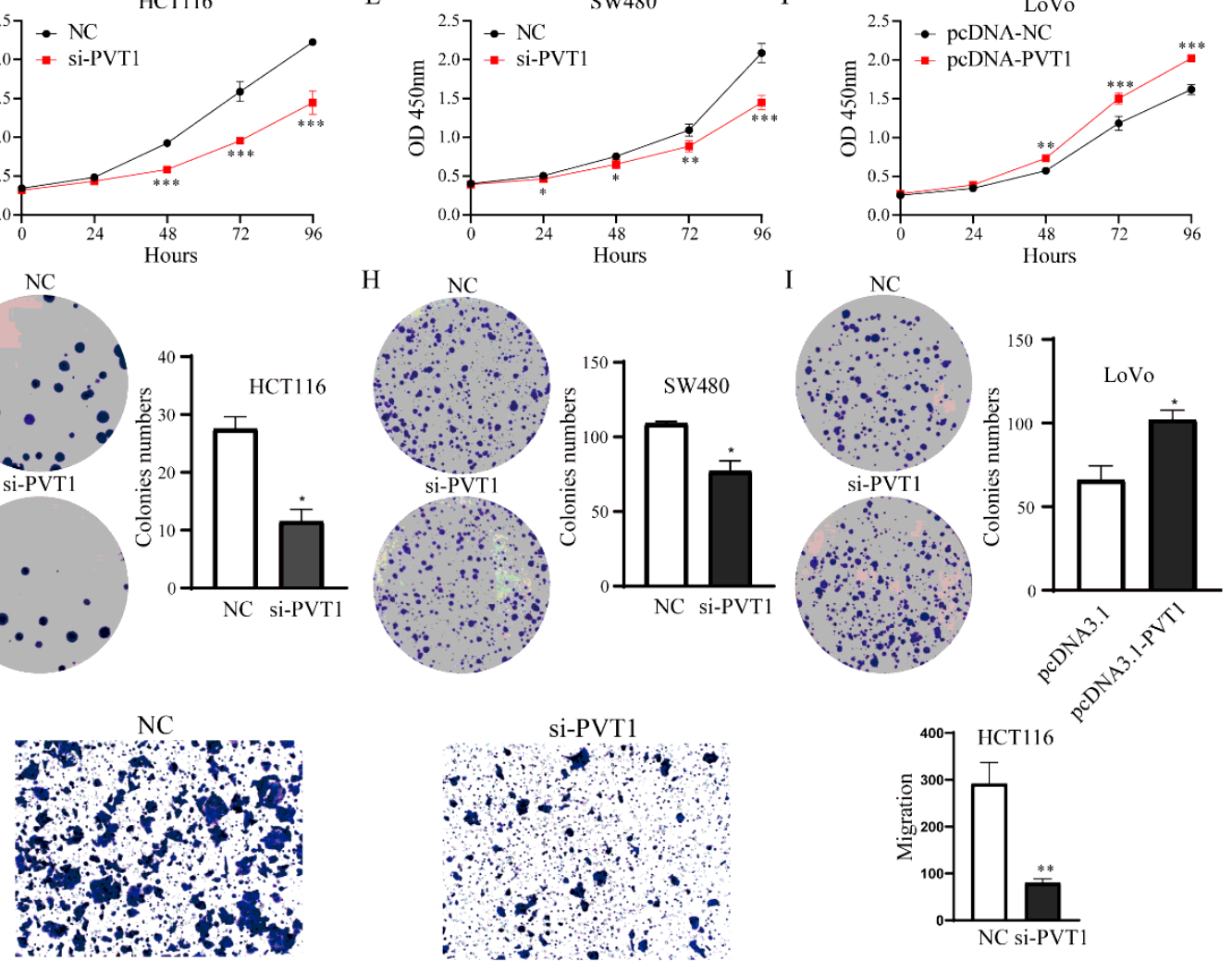

K

$\mathrm{NC}$

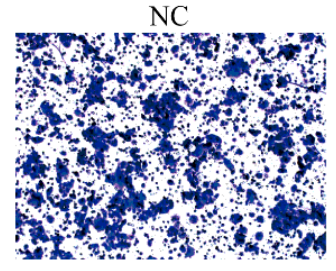

Si-PVT1
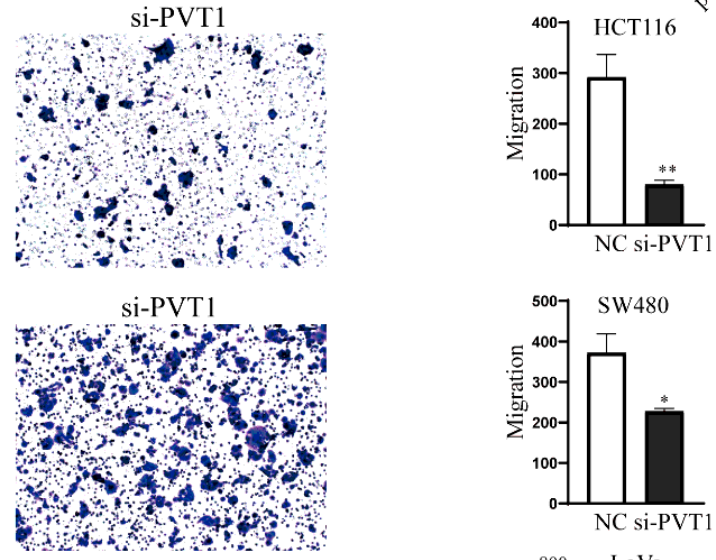

pcDNA3.1-PVT1

L
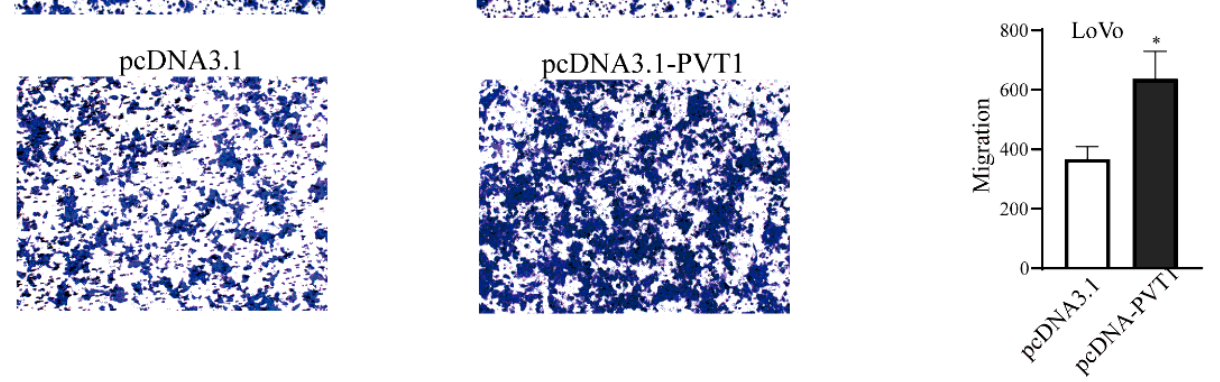


\section{Figure 2}

PVT1 promotes the proliferation and migration of CRC cellsin vitro. A The expression level of PVT1 was measured by qRT-PCR in human CRC cell lines (HCT116, SW480, SW620, LoVo) comparing to thenormal colon epithelial cell (FHC). B The knockdown efficiency of PVT1 siRNA was examined by qRT-PCR in HCT116 and SW480 cells. C The overexpression efficiency of PVT1 plasmid was examined by qRT-PCR in LoVo cells.D-F CCK-8 assayswere performed to evaluate the cell proliferation of HCT116 (D), SW480 (E) and LoVo cells (F) when downregulating or upregulating PVT1 expression. G-I Colony formation assays were performed to evaluate the cell proliferation in HCT116 (G), SW480 (H) and LoVo cells (I).J-K Transwell assays were performed to evaluate the cell migration in HCT116 (J), SW480 (K) and LoVo cells (L).*: P $<0.05, * *: P<0.01, * * *: P<0.001$. 


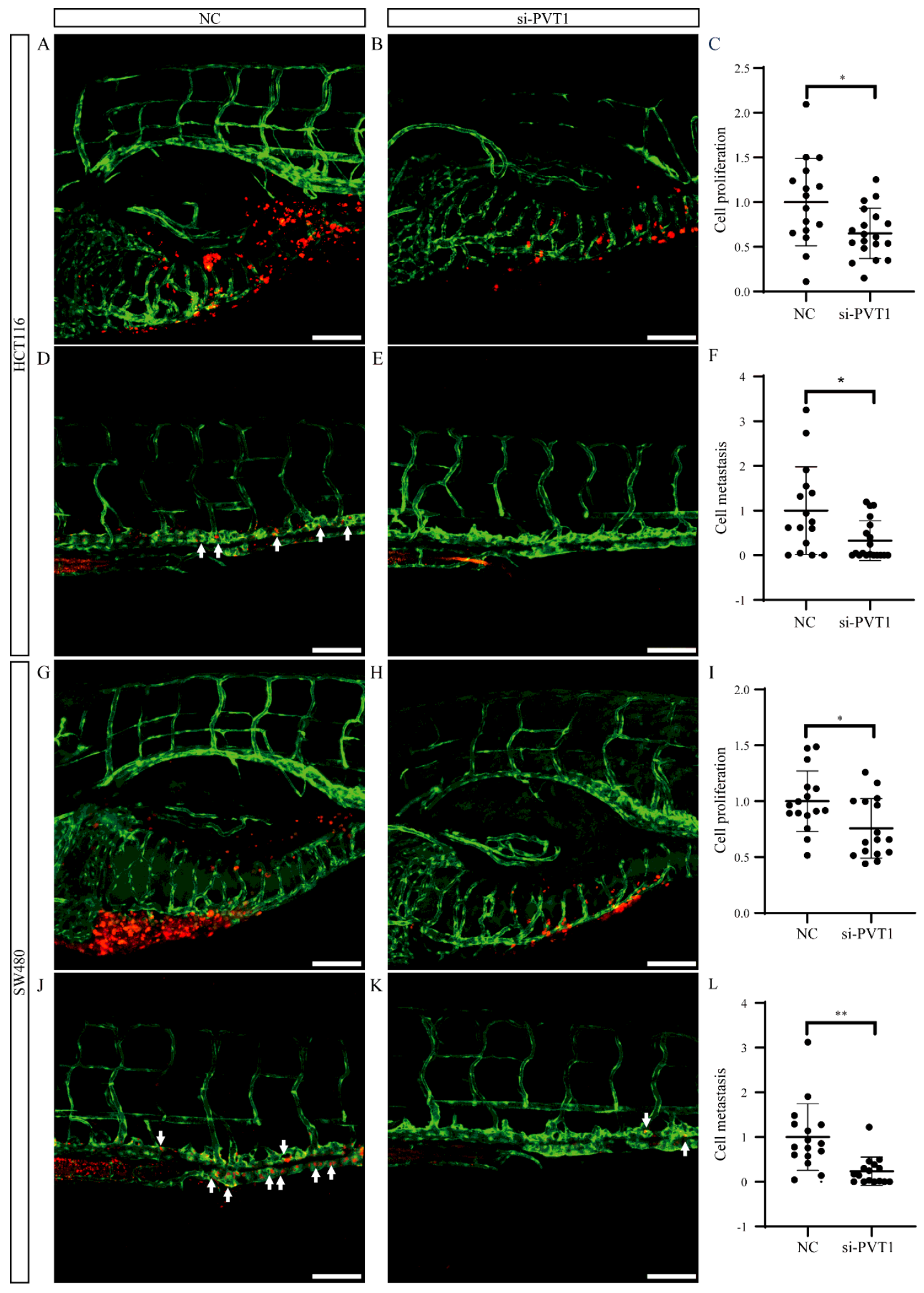

Figure 3

PVT1 knockdown in hibits the growth and metastasis of CRC cells in zebrafish xenografts. A,B The CMDil-positive signals in the yolk of zebrafish represented the proliferation of HCT116 when transfecting NC (A) or si-PVT1 (B). C Quantification of cell proliferation in zebrafish xenograft when transfecting NC or siPVT1 in HCT116 cells. D, E The CM-Dil-positive signals in the truck of zebrafish represented the metastasis of HCT116 when transfecting NC (D) or si-PVT1 (E). F Quantification of cell proliferation in 
zebrafish xenograft when transfecting NC or si-PVT1 in HCT116 cells. G-L Cell proliferation (G-I) and metastasis (J-L) in zebrafish xenograft when transfecting NC or si-PVT1 in SW480 cells. Arrows represent the metastasis CRC cells. Scale: $100 \mu \mathrm{m} . *$ : $p<0.05, * *: p<0.01$.

A
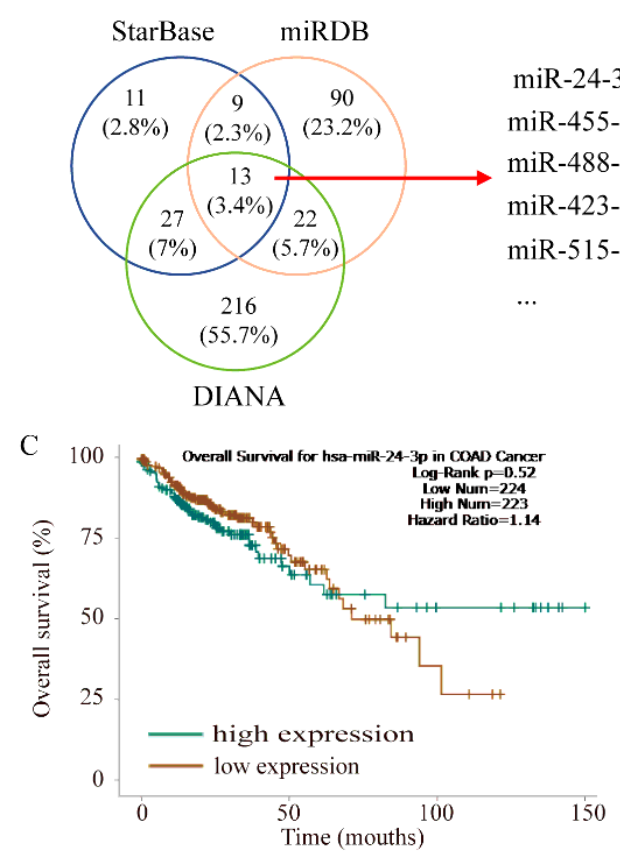

$\mathrm{E}$
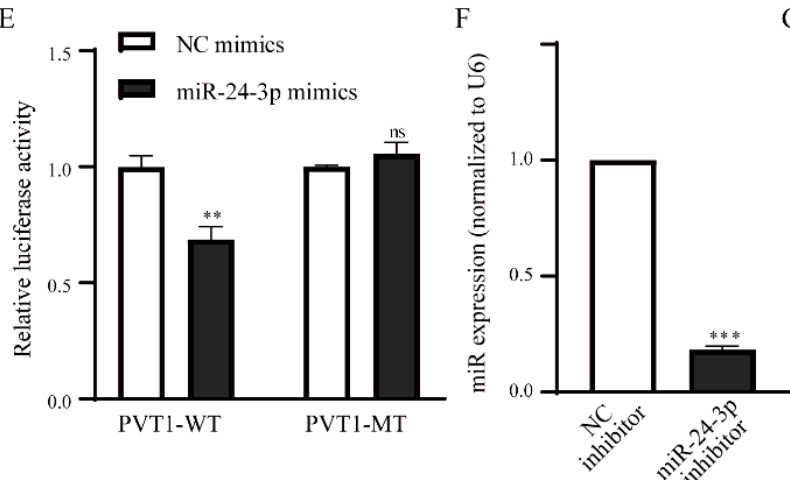

$\mathrm{H}$

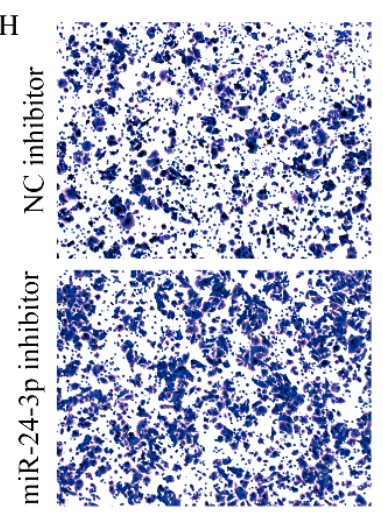

miR-24-3p

miR-455-5p miR-488-3p miR-423-5p miR-515-5p

$\mathrm{D}$

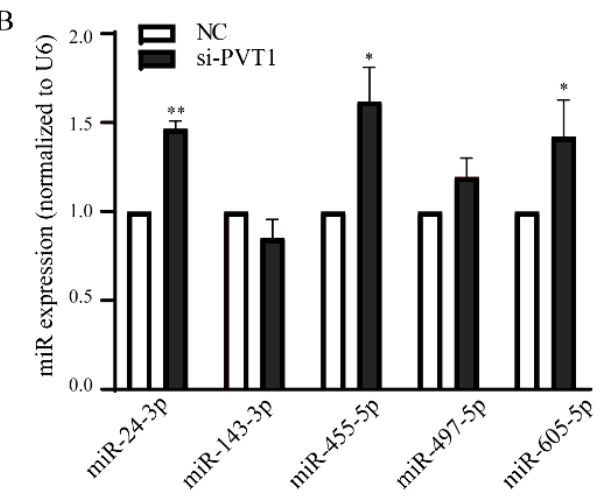

5'-UCGUUCAAGUAUUUUCUGAGCCU-3' PVT1-WT

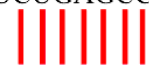

3'- GACAAGGACG-ACUUGACUCGGU-5' miR-24-3p

5'-UCGUUCAAGUAUUUUCCGGUUGU-3' PVT1-MUT

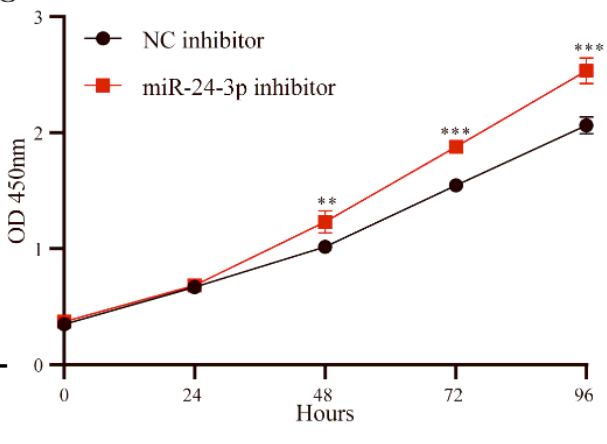

$\mathrm{J}$

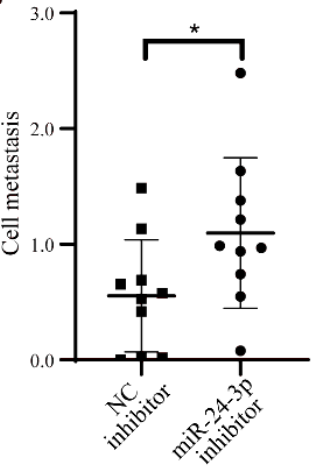

Figure 4 
miR-24-3p binds with PVT1 and negatively regulates CRC progressionin vitro and in vivo. AVenn diagram for miRNA candidates that might bind with PVT1. B The expression levels of five miRNAs was examined when PVT1 knockdown. CThe correlation between the expression levels of miR-24-3p and overall survival of CRC patients.DThe schematic diagram of potential binding site between miR-24-3p and PVT1 which was predicted by StarBase. EDual luciferase activities of PVT1-WT or PVT1-MT were examined when cotransfecting with miR-24-3p mimic. $*$ : $p<0.05$, **: $p<0.01$.F The knockdown efficiency of miR-24-3p when transfecting its inhibitor.G, $\mathbf{H}$ The proliferation and migration of CRC cells when silencing miR-24$3 p$. I, J Quantification of the growth and metastasis of CRC cells when silencing miR-24-3p by zebrafish xenografts. *: $P<0.05, * \star: ~ p<0.01, * \star *: P<0.001$.
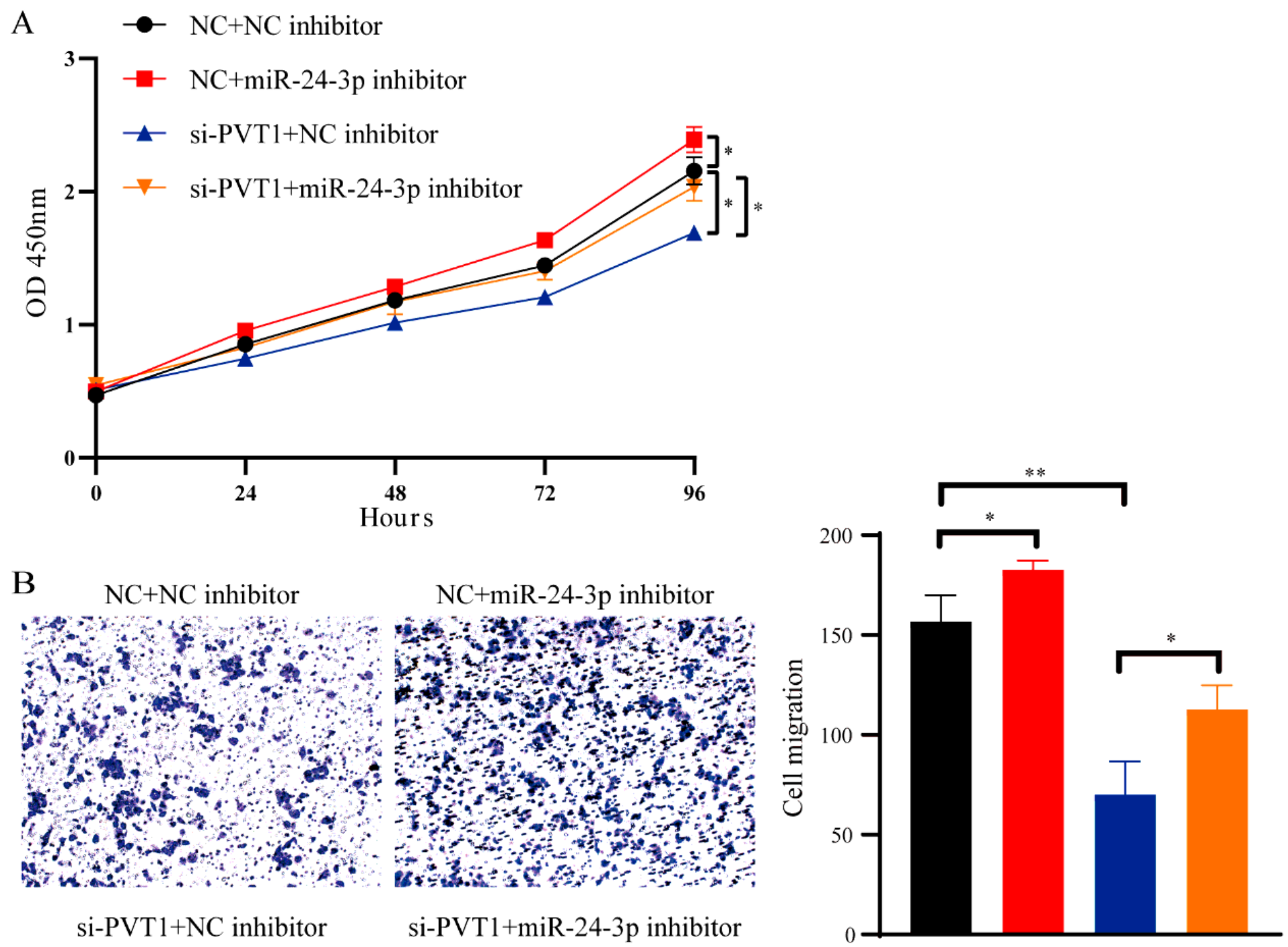

B

$\mathrm{NC}+\mathrm{NC}$ inhibitor

$\mathrm{NC}+\mathrm{miR}-24-3 \mathrm{p}$ inhibitor

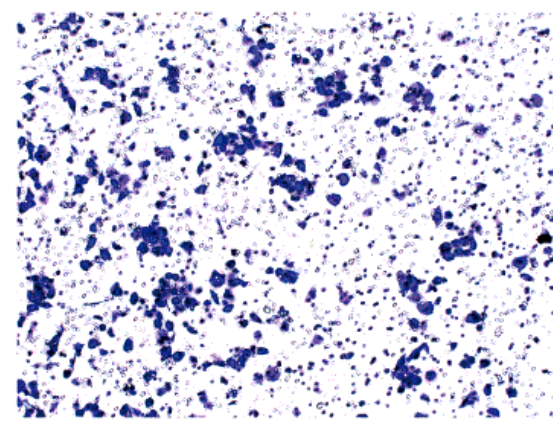

si-PVT1+NC inhibitor

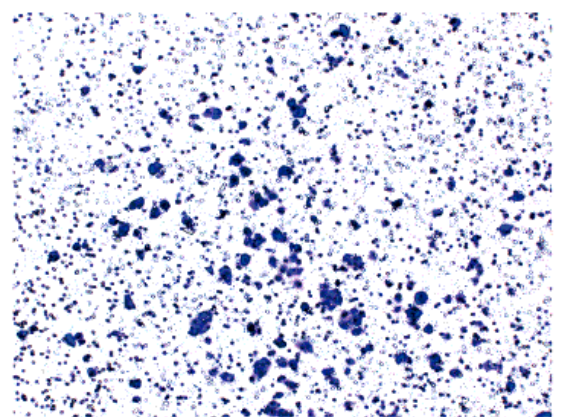

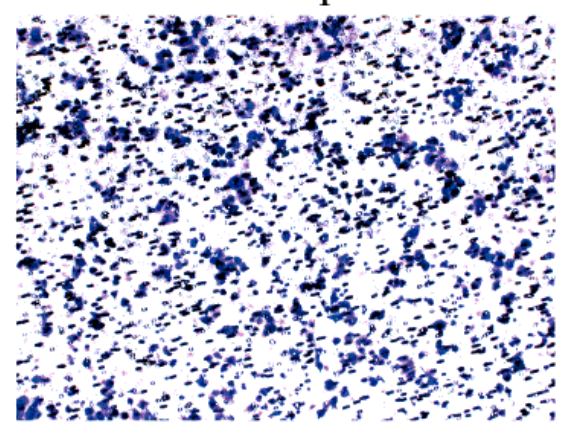

si-PVT1+miR-24-3p inhibitor

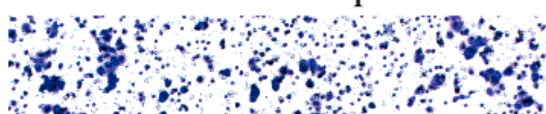

7 ato
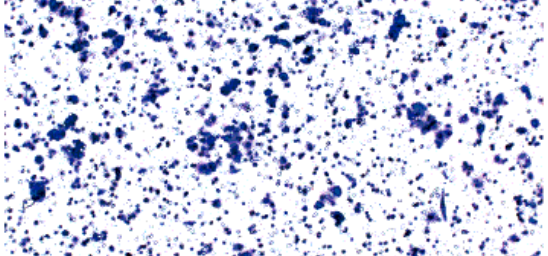

+ $-\infty, \infty, \infty$

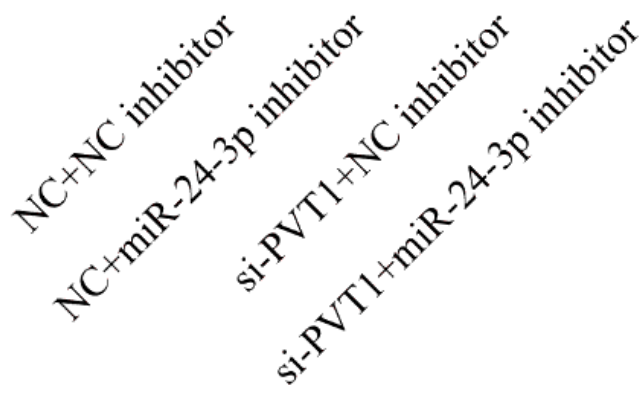


Figure 5

Knockdown of miR-24-3p rescues the progression of CRC cells which is suppressed by PVT1 silence. A The cell proliferation was evaluated by CCK-8 assay when cotransfecting miR-24-3p inhibitor and siPVT1. B The cell migration was evaluated by transwell assay when cotransfecting miR-24-3p inhibitor and si-PVT1.*: $P<0.05, * *: p<0.01$.
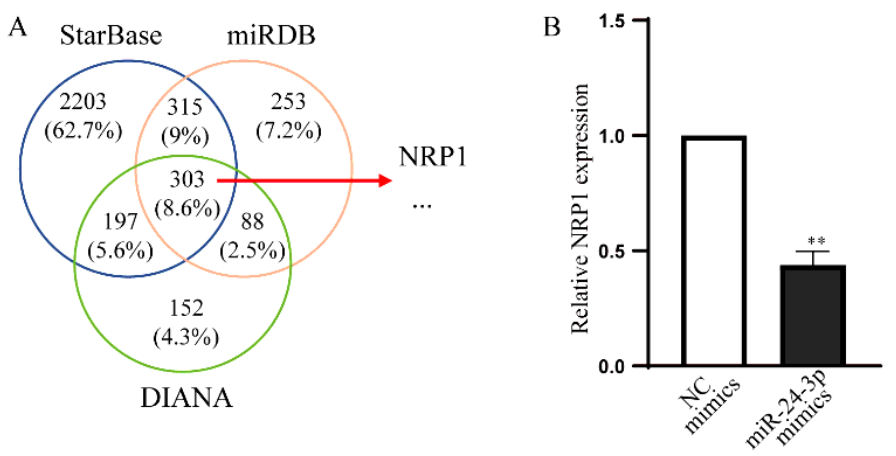

C

$\mathrm{D}$

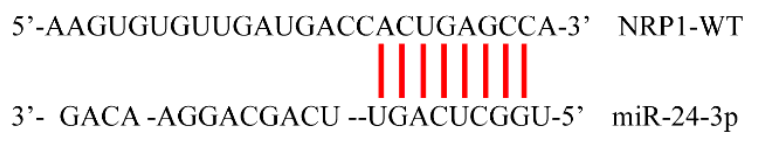

5'-AaguguguUgaugaCCAUCCGGUUG-3' PVT1-MUT

F

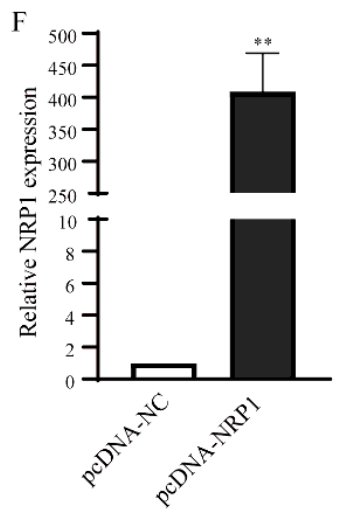

I

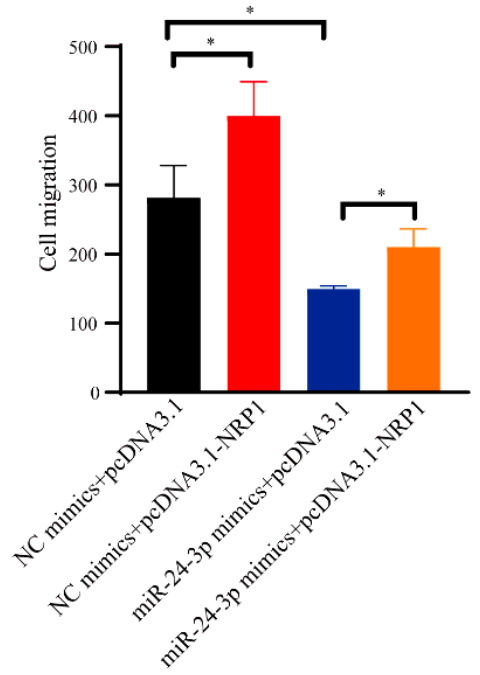

GAPDH
G

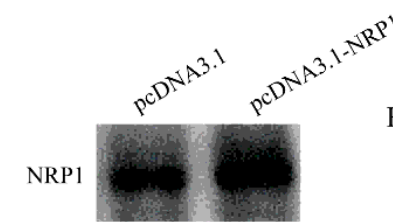

$\mathrm{H}$

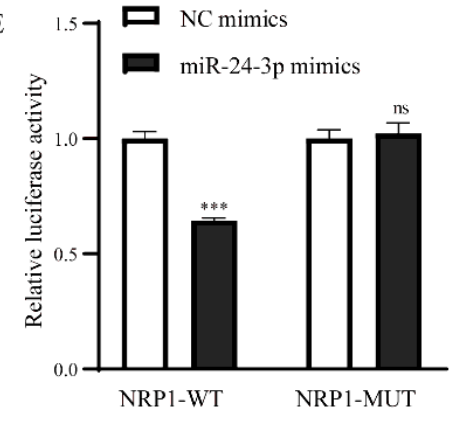

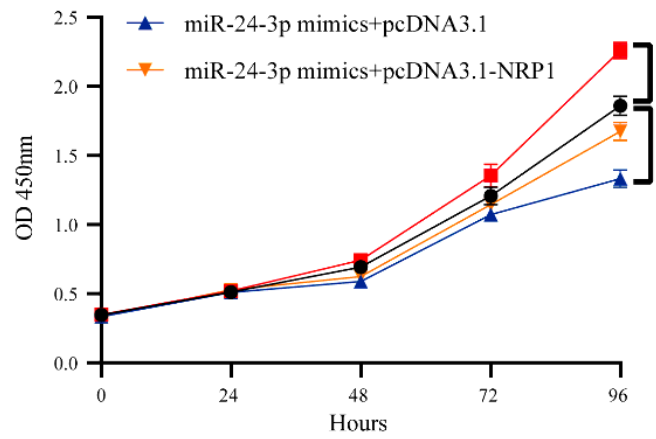

NC mimics+pcDNA3.1

NC mimics+pcDNA3.1-NRP1

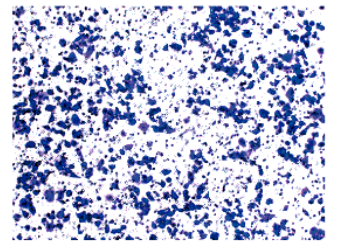

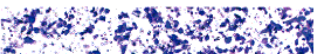
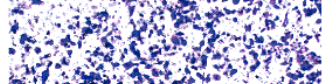

and

miR-24-3p mimics+pcDNA3.1

miR-24-3p mimics+pcDNA3.1-NRP1
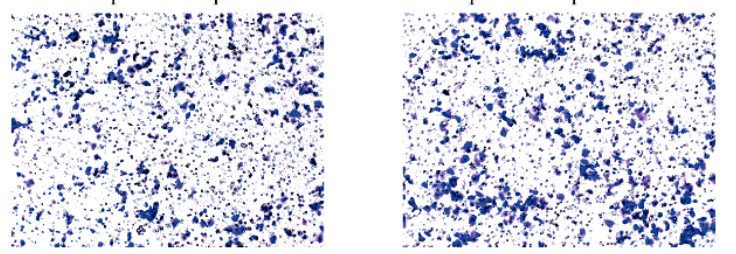


\section{Figure 6}

miR-24-3p negatively regulates CRC progression via targeting NRP1 directly. AVenn diagram for the potential target genesof miR-24-3p.B, CThe expression of NRP1 was examined by qRT-PCR and western blot when miR-24-3p over expression in CRC cells. D The schematic diagram of potential binding site between miR-24-3p and NRP1. E Dual luciferase activities of NRP1-WT and NRP1-MUT were examined when cotransfecting with miR-24-3p mimic.F, G The expression of NRP1 was measured by qRT-PCR and western blot when transfecting NRP1 overe xpression plasmid. $\mathbf{H}, \mathbf{I T}$ the proliferation $(\mathbf{H})$ and migration (I)of CRC cells were examined when cotransfecting miR-24-3p mimic and NRP1 over expression plasmid. *: $p<0.05, * *: p<0.01, * * *: P<0.001$. 

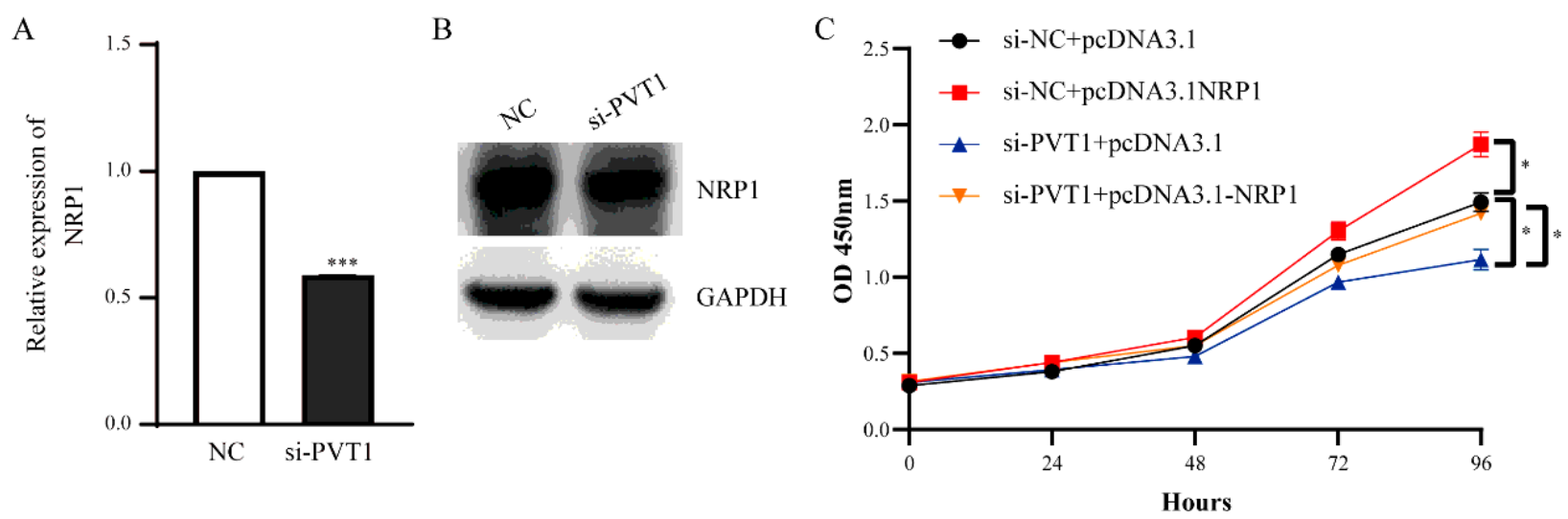

$\mathrm{D}$
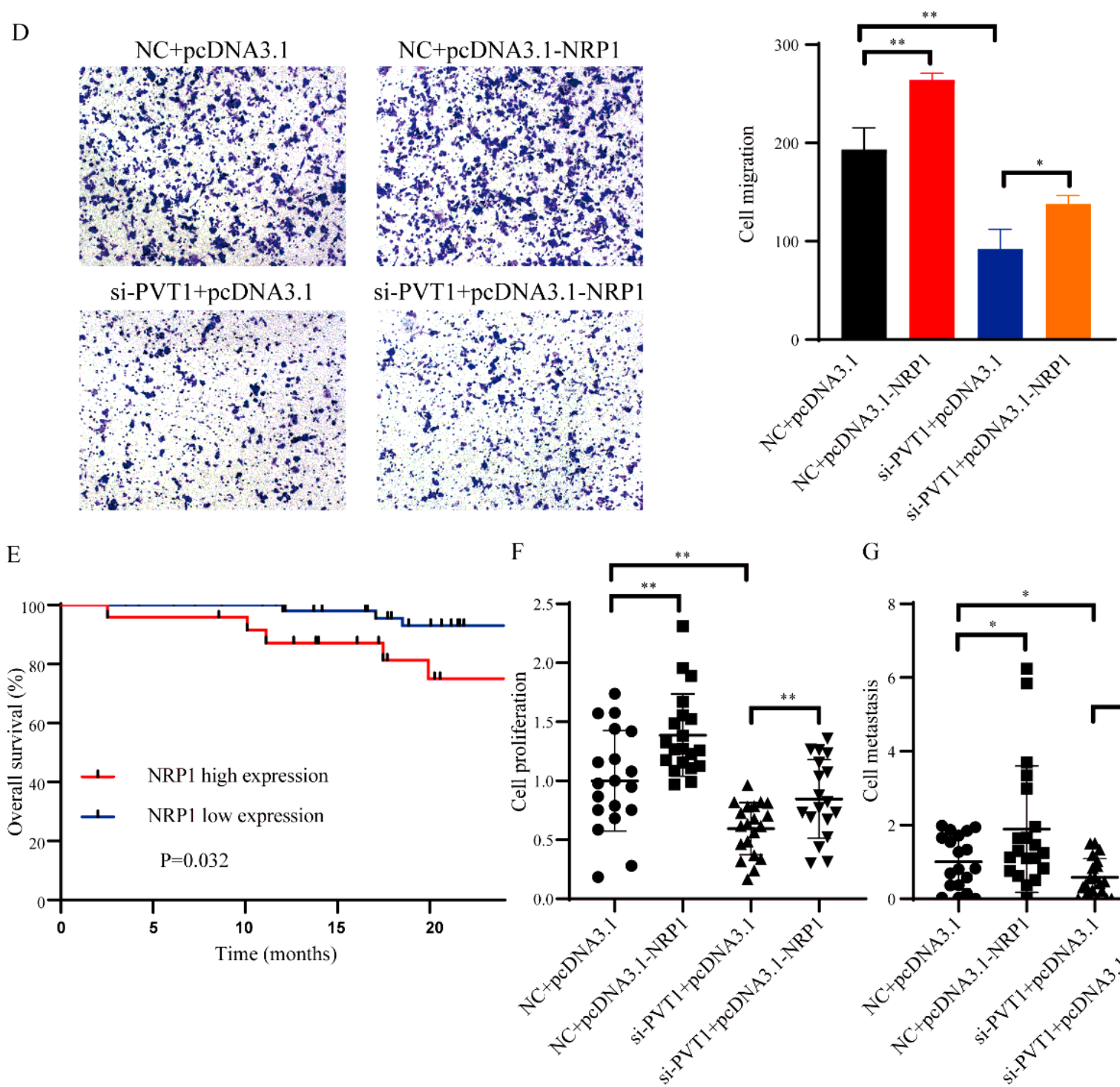

G

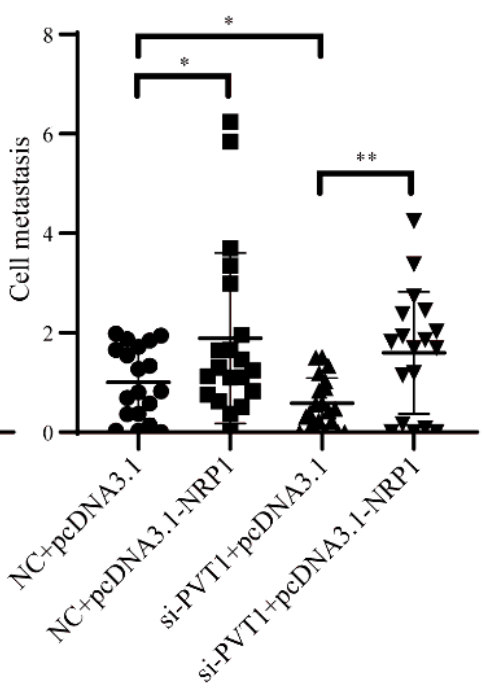

Figure 7

PVT1 regulates the progression of CRC cells via NRP1 in vitro and in vivo. A, B The expression of NRP1 was examined when silencing PVT1 in CRC cells by qRT-PCR (A) and western blot (B). C, D The proliferation (C) and migration (D) of CRC cells when cotransfecting si-PVT1 and NRP1 overexpression plasmid. EThe correlation between the expression levels of NRP1 and overall survival of CRC patients.F, G 
Quantification of the growth and metastasis of CRC cells when cotransfecting si-PVT1 and NRP1 overexpression plasmid by zebrafish xenografts. *: $p<0.05, * *$ : $p<0.01, * \star *: P<0.001$. 